\title{
Complete absence of localization in a family of disordered lattices
}

\author{
Biplab Pal $^{1}{ }^{\text {(a) }}$, Santanu K. Maiti ${ }^{2}$ (b) and Arunava Chakrabarti ${ }^{1}$ (c) \\ '1 Department of Physics, University of Kalyani, Kalyani, West Bengal-741 235, India \\ 2 Physics and Applied Mathematics Unit, Indian Statistical Institute, 203 Barrackpore Trunk Road, Kolkata-700 108, \\ India
}

PACS 71.30.th - Metal-insulator transitions and other electronic transitions

PACS 72.15.Rn - Localization effects (Anderson or weak localization)

PACS 03.75. - b - Matter waves

\begin{abstract}
We present analytically exact results to show that, certain quasi one-dimensional lattices where the building blocks are arranged in a random fashion, can have an absolutely continuous part in the energy spectrum when special correlations are introduced among some of the parameters describing the corresponding Hamiltonians. We explicitly work out two prototype cases, one being a disordered array of a simple diamond network and isolated dots, and the other an array of triangular plaquettes and dots. In the latter case, a magnetic flux threading each plaquette plays a crucial role in converting the energy spectrum into an absolutely continuous one. A flux controlled enhancement in the electronic transport is an interesting observation in the triangle-dot system that may be useful while considering prospective devices. The analytical findings are comprehensively supported by extensive numerical calculations of the density of states and transmission coefficient in each case.
\end{abstract}

Localization of electronic states in a disordered lattice, - first proposed by Anderson [1] is a problem of everlasting interest in condensed matter physics, and continues to generate intriguing features in quantum transport properties ' of randomly disordered systems. Over the years, with the improvement of fabrication and lithographic techniques 'the realm of Anderson localization [2,3] has extended beyond the electronic systems, and has encompassed tailor made artificial crystal structures, viz., the photonic [4,5], phononic [6, 7, plasmonic 8, 9] or polaritonic [10,11] lat' tices. Very recently, ultra-cold gases even allowed for the direct observation of localization of matter waves [12 14.

The pivotal result in this field is that, the electronic wave functions are known to be localized for dimensions $d \leq 2$ (the band center in the off diagonal disorder case is an exception), and even for $d>2$ for strong disorder, with an exponential decay in the envelope of the wave functions 2, 3. The result has been substantiated by meticulous analyses of various calculations related to the localization length [15, 16, density of states [17, or multi-fractality of the single particles states [18, 19. Extensive work has also been undertaken to study the in-

\footnotetext{
(a)E-mail: biplabpal@klyuniv.ac.in

(b) E-mail: santanu.maiti@isical.ac.in

(c) E-mail: arunava_chakrabarti@yahoo.co.in
}

tricacies of the single parameter scaling hypothesis - its validity [20, variance [21, or even violation [22, 23] in low dimensional systems within a tight-binding approximation, that has been subsequently consolidated by experimental measurements of conductance distribution in quasi-one dimensional gold wires 24 .

In the last decade we have come across examples, within a tight-binding description, where localizationdelocalization transitions have been observed in disordered systems. The transitions are attributed to certain special kinds of correlation in the potential profiles [25] 27] and, have unraveled the presence of discrete energy levels corresponding to extended eigenfunctions [25]. Experiments in this direction 28, 29, have substantiated the theoretical works. This led to the possibility of a spectral continuum and metal-insulator transition [26,27] in one, or quasi-one dimensional discrete systems. The idea of engineering extended states in two dimensional disordered systems 30 has also been put forward recently. In such cases the general exponentially localized character of the eigenfunctions prevail, and there is a mixed spectrum of localized and extended states (under some special correlations as discussed above).

In the present letter, we present examples where even in a disordered arrangement of scatterers, delocalization 
of electronic states can occur throughout the energy spectrum, making it an absolutely continuous one. This happens for a class of quasi one-dimensional systems when special correlations are introduced among the numerical values of the parameters in the Hamiltonian describing them. Two distinct cases are presented. In the first case, we consider a diamond network of four atomic like sites placed at random in a host lattice of isolated 'atoms' (which may be thought of as single level quantum dots). The system is described by a tight-binding Hamiltonian. The on-site potential at every lattice point is taken to be

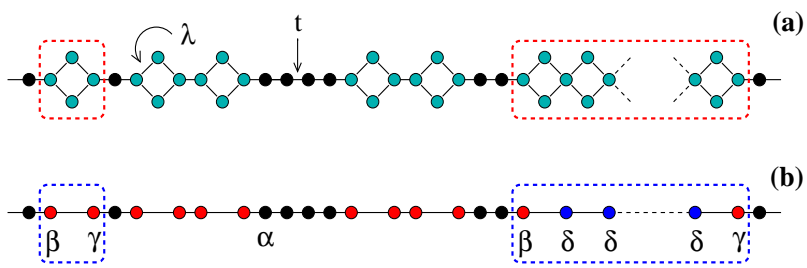

Fig. 1: (Color online). (a) A typical realization of an array of isolated atomic sites (marked as black circles) and the diamond shaped quadruplets (in isolation, and in clusters, and marked by light blue circles). (b) The renormalized $1-d$ chain, where the isolated dots remain un-renormalized, while the diamond clusters are renormalized into $\beta-\gamma$ doublets, or a sequence of $\beta-\delta^{n}-\gamma$. The hopping integral along the major axis (backbone) is $t$, while it is $\lambda$ along the edges of a diamond. All on-site potentials are equal.

a constant, while the nearest-neighbor hopping integral along the arms of the diamond $(\lambda)$ differ from that along the back bone $(t)$, where it is assumed to be constant (see fig. (1). There is no positional correlation, short range or long range in the conventional sense $25 \mid 26$. We show that, for a particular relationship between the nearest-neighbor hopping integrals, the infinite diamond-dot (DD) chain will yield an absolutely continuous spectrum, and that, the two terminal transmission coefficient will be unity, irrespective of the energy of the electron.

In the second case, the diamond network is replaced by a triangular one leading to an infinite triangle-dot (TD) chain. Each triangular plaquette is threaded by an external magnetic flux. The parameters of the Hamiltonian of this TD chain are same on that in the DD array. It will be shown that in this case, apart from the resonance condition satisfied by $\lambda$ and $t$ as in the DD array, the external flux needs to be fixed at a special value to make the spectrum absolutely continuous. Thus, in this example, the general localized character of the spectrum and the low electronic transmission in an otherwise disordered arrangement of scatterers can be enhanced by an external magnetic field.

In this context, it should be mentioned that the question of inducing a whole continuum of extended states in a disordered array of potentials has previously been addressed by Rodriguez and Cervero 31 using a continuous version of the Schrödinger equation and with a special kind of po- tential profile. In our case, we focus on a discrete lattice model, and the disorder is introduced in the geometrical arrangement of the atomic sites along a major axis. The coordination numbers of the lattice points range between 2,3 and 4 in every case, and are distributed in a completely uncorrelated, random fashion. This aspect, to the best of our knowledge, has not been discussed in the literature before. We now discuss the two cases separately.

The diamond-dot (DD) system. - Spinless, noninteracting electrons on the system are described by the Hamiltonian,

$$
H_{S}=\epsilon \sum_{i} d_{i}^{\dagger} d_{i}+\sum_{\langle i j\rangle} t_{i j}\left[d_{i}^{\dagger} d_{j}+\text { h.c. }\right]
$$

where, $\epsilon$ is the constant on-site potential, the nearestneighbor hopping integral $t_{i j}=t$ along the backbone, and $t_{i j}=\lambda$ along an edge of the diamond. $d_{i}^{\dagger}\left(d_{i}\right)$ represents the creation (annihilation) operator. The Schrödinger equation, written equivalently in the form of the difference equation, $(E-\epsilon) \psi_{i}=\sum_{j} t_{i j} \psi_{j}$ allows us to decimate out the vertices of the diamond networks to map the original chain on to an effective one dimensional chain [fig. 1(b)] of four sites $\alpha, \beta, \gamma$ and $\delta$ with on-site potentials

$$
\begin{aligned}
& \epsilon_{\alpha}=\epsilon \\
& \epsilon_{\beta}=\epsilon_{\gamma}=\epsilon+2 \lambda^{2} /(E-\epsilon) \\
& \epsilon_{\delta}=\epsilon+4 \lambda^{2} /(E-\epsilon) .
\end{aligned}
$$

There is a binary distribution of hopping integrals along the chain now. These are the original $t$, and the effective coupling

$$
\tau=2 \lambda^{2} /(E-\epsilon)
$$

arising out of the renormalization of the diamond network.

Using the difference equation the amplitudes of the wave function at the neighboring sites along the effective one dimensional chain can be related by the $2 \times 2$ transfer matrices,

$$
\left(\begin{array}{c}
\psi_{n+1} \\
\psi_{n}
\end{array}\right)=\left(\begin{array}{cc}
\frac{E-\epsilon_{n}}{t_{n, n+1}} & -\frac{t_{n, n-1}}{t_{n, n+1}} \\
1 & 0
\end{array}\right)\left(\begin{array}{c}
\psi_{n} \\
\psi_{n-1}
\end{array}\right)(4)
$$

A look at the fig. 1(b) will make it obvious that there are four kinds of transfer matrices, viz, $M_{\alpha}, M_{\beta}, M_{\gamma}$ and $M_{\delta}$, which will differ in their matrix elements, depending on the respective on-site potentials and the nearest-neighbor hopping integrals. From the arrangement of the diamonds and the isolated sites in the original DD chain it can be appreciated that the the wave function at a far end of the chain can be determined if one evaluates the product of the unimodular matrices $M_{\alpha}, M_{\gamma \beta}=M_{\gamma} \cdot M_{\beta}$ and $M_{\gamma \delta \beta}=$ $M_{\gamma} \cdot M_{\delta} \cdot M_{\beta}$, or $M_{\gamma \delta^{n} \beta}=M_{\gamma} \cdot M_{\delta}^{n} \cdot M_{\beta}$ sequenced in the desired random fashion.

The central result of this communication is that, the commutators $\left[M_{\alpha}, M_{\gamma \beta}\right]=0,\left[M_{\alpha}, M_{\gamma \delta \beta}\right]=0$, and 
$\left[M_{\gamma \beta}, M_{\gamma \delta \beta}\right]=0$ irrespective of the energy $E$ of the electron whenever we choose $\lambda=t / \sqrt{2}$. To see this explicitly we display the diagonal and the off-diagonal elements of the above three commutators. These are,

$$
\begin{aligned}
& {\left[M_{\alpha}, M_{\gamma \beta}\right]_{11}=\left[M_{\alpha}, M_{\gamma \beta}\right]_{22}=0} \\
& {\left[M_{\alpha}, M_{\gamma \beta}\right]_{12}=\left[M_{\alpha}, M_{\gamma \beta}\right]_{21}=\frac{(E-\epsilon)\left(t^{2}-2 \lambda^{2}\right)}{2 t \lambda^{2}}} \\
& {\left[M_{\gamma \beta}, M_{\gamma \delta \beta}\right]_{11}=\left[M_{\gamma \beta}, M_{\gamma \delta \beta}\right]_{22}=0} \\
& {\left[M_{\gamma \beta}, M_{\gamma \delta \beta}\right]_{12}=\left[M_{\gamma \beta}, M_{\gamma \delta \beta}\right]_{21}=\frac{(E-\epsilon)\left(t^{2}-2 \lambda^{2}\right)}{2 t \lambda^{2}}} \\
& {\left[M_{\alpha}, M_{\gamma \delta \beta}\right]_{11}=\left[M_{\alpha}, M_{\gamma \delta \beta}\right]_{22}=0} \\
& {\left[M_{\alpha}, M_{\gamma \delta \beta}\right]_{12}=\left[M_{\alpha}, M_{\gamma \delta \beta}\right]_{21}=\frac{(E-\epsilon)\left(t^{2}-2 \lambda^{2}\right) \mathcal{F}}{4 t \lambda^{4}}}
\end{aligned}
$$

where, $\mathcal{F} \equiv \mathcal{F}(E, \epsilon, \lambda)=\left[(E-\epsilon)^{2}-4 \lambda^{2}\right]$.

An interesting observation is that, in an infinite array of diamonds and dots, without any restriction on randomness, there can be isolated diamonds (equivalent to a $\beta-\gamma$ pair), or a sequence of $n+1$ diamonds (equivalent to a sequence of clusters $\beta-\delta^{n}-\gamma$ ). The total transfer matrix for the latter cluster can be written as, $M_{\gamma \delta n \beta}=U_{n-1}(x) M_{\gamma \delta \beta}-U_{n-2}(x) M_{\gamma \beta}$, where, $U_{n}(x)$ is the $n$-th order Chebyshev polynomial of the second kind and $x=\operatorname{Tr}\left(M_{\delta}\right) / 2$. It immediately becomes obvious that $\left[M_{\alpha}, M_{\gamma \delta^{n} \beta}\right]=0$ for any value of $n$, as $M_{\alpha}$ commutes with both $M_{\gamma \beta}$ and $M_{\gamma \delta \beta}$. In addition to this, the commutation of $M_{\gamma \beta}$ and $M_{\gamma \delta^{n} \beta}$ also becomes obvious. This implies that, with $\lambda=t / \sqrt{2}$ the amplitude of the wave function or its phase at any lattice point on the renormalized chain [fig. 1(b)] in the actual randomly disordered array of diamond and dots will be indistinguishable from that in a perfectly periodic arrangement of these clusters. This happens independent of the energy $E$ of the electron, that is, throughout the energy spectrum, and is true for any kind of disordered arrangement that one can build using a dot, isolated diamonds and an array of any $n$ number of diamonds in juxtaposition. The wave functions as result, will have to be of a perfectly extended, Bloch-like character.

The energy spectrum in the above case is absolutely continuous within the range $[\epsilon-2 t, \epsilon+2 t]$, and the local density of states (LDOS) at any nodal point $\alpha, \beta, \gamma$ or $\delta$ on the renormalized lattice resembles that of a perfectly ordered lattice of identical sites with on-site potential $\epsilon$ and nearest neighbor hopping integral $t$. We have extensively verified this, though present only the average density of states to save space. Nevertheless, the fact that it should be the reality, can be tested by observing that under the condition $\lambda=t / \sqrt{2}$, the on-site potentials on the renormalized chain in fig. 1(b) are, $\epsilon_{\alpha}=\epsilon, \epsilon_{\beta}=\epsilon_{\gamma}=\epsilon+t^{2} /(E-\epsilon)$, and $\epsilon_{\delta}=\epsilon+2 t^{2} /(E-\epsilon)$. The nearest-neighbor hopping matrix elements turn out to be $t$ (unchanged value) and $\tau=t^{2} /(E-\epsilon)$. Most interestingly, identical values can be obtained by beginning with a perfectly periodic chain with constant on-site potential $\epsilon$ and nearest-neighbor hopping $t$, and by arbitrarily decimating sites so as to reproduce the same disordered pattern as the original chain. The process is illustrated in fig. 2. Now, the lattice in fig. 2 is

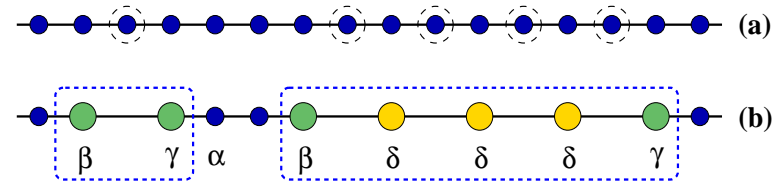

Fig. 2: (Color online). (a) A perfectly periodic array of identical atomic sites (dots). (b) The renormalized lattice is obtained by decimating the encircled dots in (a). This generates a chain where the $\beta, \gamma$ and $\delta$ sites and the nearest-neighbor hopping integrals have precisely those values as obtained in the disordered DD array by setting $\lambda=t / \sqrt{2}$.

a perfectly periodic one, and no matter how we decimate sites, the LDOS at any site will be that of a periodic chain with van Hove singularities marking the band edges. This is what makes the LDOS at any site on the backbone of our original system in fig. 1(b) indistinguishable from that of an ordered chain of atoms.

It is also important to look at the individual spectra of three subsystems comprising of $\alpha, \beta \gamma$ and $\beta \delta \gamma$ clusters. The spectrum of a 'pure' $\alpha$-lattice consists of extended eigenstates spanning an energy interval $[\epsilon-2 t, \epsilon+2 t]$. If we start out with an ordered array of $\beta \gamma$ clusters, it is simple to show that the LDOS, say, at the $\beta$ site is,

$$
\rho_{\beta}^{(\beta \gamma)}=\frac{1}{\pi} \frac{(E-\epsilon)^{2}-2 \lambda^{2}}{\left(16 t^{2} \lambda^{4}-(E-\epsilon)^{2}\left[(E-\epsilon)^{2}-t^{2}-4 \lambda^{2}\right]^{2}\right)^{1 / 2}}
$$

Similarly, in an ordered array of $\beta \delta \gamma$ clusters, the LDOS at the $\beta$ site is of the form,

$$
\rho_{\beta}^{(\beta \delta \gamma)}=\frac{1}{\pi} \frac{\mathcal{A}(E, \lambda, \epsilon, t)}{\mathcal{B}(E, \lambda, \epsilon, t)}
$$

where, $\mathcal{A}(E, \lambda, \epsilon, t)=E^{4}-4 E^{3} \epsilon+\epsilon^{4}-6 \epsilon^{2} \lambda^{2}+4 \lambda^{4}+$ $6 E^{2}\left(\epsilon^{2}-\lambda^{2}\right)-4 E\left(\epsilon^{3}-3 \epsilon \lambda^{2}\right)$ and $\mathcal{B}(E, \lambda, \epsilon, t)=\left(64 t^{2} \lambda^{8}-\right.$ $(E-\epsilon)^{2}\left[E^{4}-4 E^{3} \epsilon+\epsilon^{4}+E^{2}\left(6 \epsilon^{2}-t^{2}-8 \lambda^{2}\right)+4 \lambda^{2}\left(3 \lambda^{2}+\right.\right.$ $\left.\left.\left.t^{2}\right)-\epsilon^{2}\left(8 \lambda^{2}+t^{2}\right)+2 E \epsilon\left(8 \lambda^{2}+t^{2}-2 \epsilon^{2}\right)\right]^{2}\right)^{1 / 2}$

In each case, on substituting $\lambda=t / \sqrt{2}$, the LDOS at the $\beta$ site reduces to the form,

$$
\rho_{\beta}^{(\beta \gamma)}=\rho_{\beta}^{(\beta \delta \gamma)}=\frac{1}{\pi} \frac{1}{\sqrt{4 t^{2}-(E-\epsilon)^{2}}}
$$

which is exactly the LDOS at any $\alpha$ site of a pure, infinite $\alpha$-chain. Thus, the resonance condition, apart from making the matrices commute, ensures that the bands of the respective sub-systems overlap at least within the energy 
interval $[\epsilon-2 t, \epsilon+2 t]$. In fact, this spectral overlap is essential to ensure complete transparency in this energy range together with the extendedness of the wave functions.

To end this part of the discussion we would like to mention that resonant (extended) eigenstates arising out of the commutivity of transfer matrices has been addressed also in relation to an array of quantum wells [32, and a distribution of potentials of Pöschl-Teller type [33] using a continuous version of the Schrödinger equation. The difference with the present case is that, here one can have commutators independent of the energy of the electron.

To obtain the energy spectrum and the transmission probability numerically we use a Green's function formal-
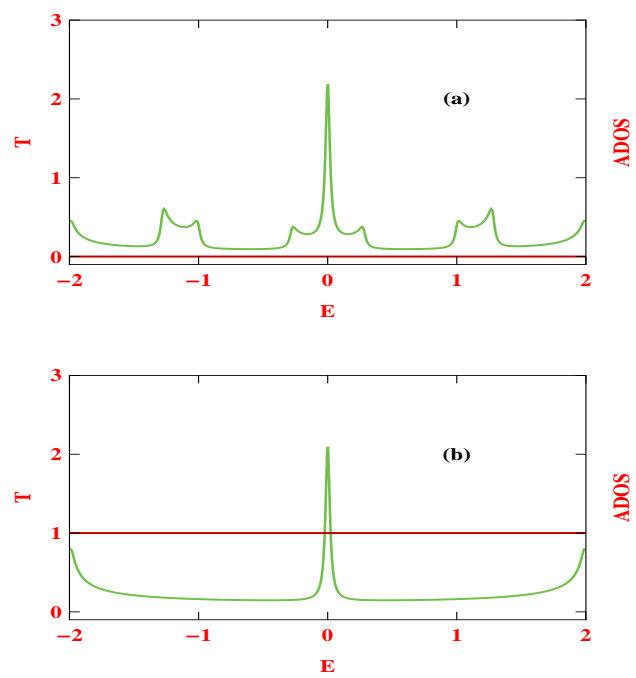

Fig. 3: (Color online). Transmission probability $T$ (red color) and Average Density of States (ADOS) (green color) as a function of energy $E$ for a completely disordered array of 100 diamonds and 100 dots. The results are averaged over 50 disorder configurations. In the non-resonant case (a) we have chosen $\lambda=0.3$, while $\lambda=1 / \sqrt{2}$ in (b) where we have complete transparency. Other parameters are, $t=1=t_{0}, \epsilon_{i}=0$ at every site, $\tau_{L}=\tau_{R}=1$, and $\epsilon_{0}=0$ in the leads.

ism 34. Keeping in mind a possible experimental realization of the system, we clamp a finite sized system of $N$-sites between two ideal semi-infinite electrodes (the left and the right electrodes) making an electrode-systemelectrode (ESE) bridge. The electrodes are described by the Hamiltonian,

$$
H_{0}=\sum_{i} \epsilon_{0} c_{i}^{\dagger} c_{i}+\sum_{<i j>} t_{0}\left(c_{i}^{\dagger} c_{j}+c_{j}^{\dagger} c_{i}\right)
$$

where different parameters correspond to their usual meaning. The couplings between the left $(\mathrm{L})$ and the right $(\mathrm{R})$ electrodes and the system $(\mathrm{S})$ are given by, $H_{L S}=\tau_{L} c_{0}^{\dagger} d_{1}+$ h.c. , and $H_{R S}=\tau_{R} d_{N}^{\dagger} c_{N+1}+$ h.c., so that, the full Hamiltonian of the ESE bridge is given by, $H=H_{S}+H_{0}+H_{L S}+H_{R S}$. The average density of states
(ADOS) of the ESE bridge is given by,

$$
\rho_{a v}=-\frac{1}{N \pi} \operatorname{Im}[\operatorname{Tr}(G)]
$$

where, $G=(E-H+i \eta)^{-1}$, with $\eta \rightarrow 0$.

In terms of the Green's function of the system and its coupling to the side-attached electrodes, the transmission probability can be written in the form 34,

$$
T=\operatorname{Tr}\left[\Gamma_{L} G_{S}^{r} \Gamma_{R} G_{S}^{a}\right]
$$

where, $\Gamma_{L}$ and $\Gamma_{R}$ describe the coupling of the system to the left and right electrodes, respectively. Here, $G_{S}^{r}$ and $G_{S}^{a}$ are the retarded and advanced Green's functions, respectively, of the system including the effects of the electrodes.

The full system is partitioned into sub-matrices corresponding to the individual sub-systems and the Green's function for the disordered sample is effectively written as,

$$
G_{S}=\left(E-H_{S}-\Sigma_{L}-\Sigma_{R}\right)^{-1}
$$

where, $\Sigma_{L}$ and $\Sigma_{R}$ are the self-energies due to coupling of the system to the left and right electrodes, respectively [34. All information of the coupling are included in these two self-energies.

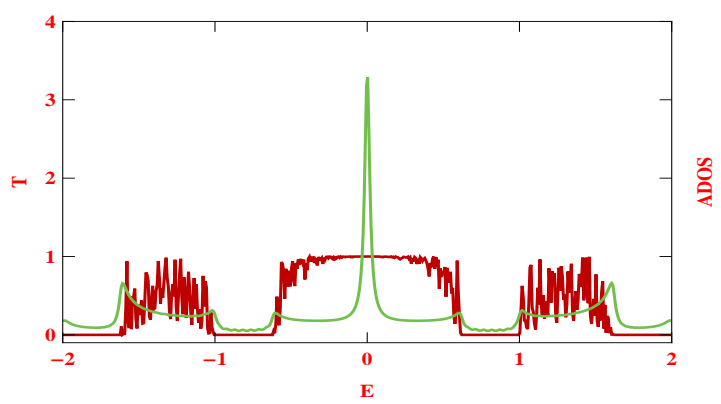

Fig. 4: (Color online). Transmission probability $T$ (red color) and ADOS (green color) as a function of energy $E$ for the diamond-dot chain away from the resonant case. The results averaged over 50 disorder configurations have been presented. 100 diamonds with 100 dots have been taken with $\lambda=0.98499$, a forty percent deviation from the resonance value of $1 / \sqrt{2}$. Other parameters are the same as in the earlier figures.

In fig. 3 we show the ADOS and the transmission coefficient of a random sequence of 100 diamonds and 100 dots. Our previous analytical argument for the infinite system is perfectly corroborated in these diagrams. Complete localization of all the eigenstates, corresponding to zero transmission, is observed [fig. [3(a)] as long as $\lambda$ is set quite arbitrarily. The scenario changes as soon as the value of $\lambda$ is set equal to the resonant value of $1 / \sqrt{2}$ (with $t=1$ ). We now see an absolutely continuous energy spectrum between $E= \pm 2 t$, the central peak at $E=0$ being the contributions from the top and bottom sites of the diamond plaquettes, and the transmission coefficient becomes equal to unity irrespective of the energy of the electron. 
Keeping in mind an experimental realization of such a finite size sample, we have carefully studied the effect of a possible deviation in the value of $\lambda$ from its ideal 'resonance value' of $1 / \sqrt{2}$. The continuity of the spectrum stands out to be a robust result even when the deviation is substantial. In fig. 4 we show the ADOS and the transmission coefficient for a $40 \%$ deviation in the value of $\lambda$ from its resonant value. The transmission spectrum is seen to be split into three continuous sub-bands of high transmission. The central sub-band still retains its continuous character. The reason is, the commutators [Eq. (5) - (77)] vanish whenever $E=\epsilon$, even for an arbitrary value of $\lambda$. Then the band center corresponds to an extended state. The transmission coefficient will be almost unity for a finite sized system even when we are off-resonance.

The triangle-dot (TD) system. - We now move to our second example where, we have a disordered array of triangular plaquettes and isolated 'dots' [fig. 5)(a)]. Each arm of a triangle is assigned a hopping integral $\lambda$, and every triangle is threaded by a magnetic flux $\Phi$. The hopping integral in between triangles and dots is equal to $t$ and the on-site potential at every lattice point is $\epsilon$ as before. Once again, decimating the vertices of every triangle

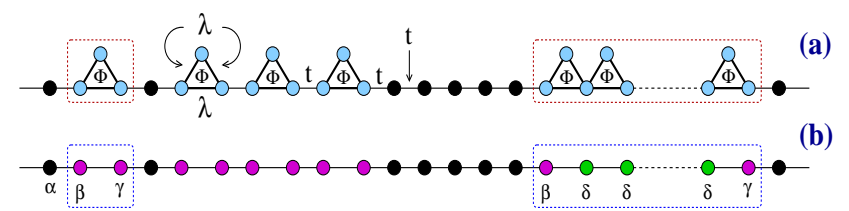

Fig. 5: (Color online). (a) A disordered arrangement of triangular plaquettes threaded by a magnetic flux $\Phi$, and dots (marked by black circles). The inter-atomic hopping integral between the sites of the triangular plaquette is $\lambda$, and the hopping integral has a value $t$ between two consecutive triangles or dots, or in between a triangle and a dot. (b) The effective 1-d chain obtained by renormalizing the lattice in (a).

we arrive at an effective one dimensional chain [fig. [5](b)] where time reversal symmetry is broken across the 'bonds' joining the base atoms (red colored points) of a triangle. The sites residing at the 'left' and the 'right' corners of a triangle will be called the $\beta$ - and $\gamma$-sites with an effective on-site potential given by,

$$
\epsilon_{\beta}=\epsilon_{\gamma}=\epsilon+\frac{\lambda^{2}}{E-\epsilon}
$$

The hopping integral connecting a $\beta-\gamma$ pair is now given by,

$$
t_{F}=\frac{\lambda^{2} e^{2 i \theta}}{E-\epsilon}+\lambda e^{-i \theta}
$$

when an electron 'hops' from a $\beta$ to a $\gamma$ site, and $t_{B}=t_{F}^{*}$ while hopping 'backward' - a consequence of broken time reversal symmetry. In the above equation $\theta=2 \pi \Phi / 3 \Phi_{0}$ where, $\Phi_{0}=h c / e$ is the fundamental flux quantum.

One can now look for a possible commutation of the same matrices, viz., $M_{\alpha}, M_{\gamma \beta}$ and $M_{\gamma \delta \beta}$ in the spirit of the DD case in earlier section. In every case, the diagonal elements of the commutators turn out to be zero, and the off diagonal elements are given by,

$$
\begin{aligned}
& {\left[M_{\alpha}, M_{\gamma \beta}\right]_{12}=-e^{i \theta} \frac{\chi}{\lambda t \Delta}} \\
& {\left[M_{\alpha}, M_{\gamma \delta \beta}\right]_{12}=-e^{2 i \theta} \frac{(E-\epsilon)^{2}-2 \lambda^{2}}{\lambda^{2} t \Delta^{2}} \chi} \\
& {\left[M_{\gamma \beta}, M_{\gamma \delta \beta}\right]_{12}=-\frac{(E-\epsilon) e^{3 i \theta}+\lambda}{\lambda t \Delta^{2}} \chi}
\end{aligned}
$$

where, $\Delta=E-\epsilon+\lambda \exp 3 i \theta$ and,

$$
\chi=2 \lambda^{3} \cos 3 \theta-(E-\epsilon)\left(t^{2}-2 \lambda^{2}\right)
$$

The $(2,1)$ element of every commutator matrix is equal to its $(1,2)$ element.

A look at Eq. (18) immediately reveals that, selecting $\Phi=\Phi_{0} / 4$ and $\lambda=t / \sqrt{2}$ makes every commutator vanish. This is the desired result. The entire randomly disordered array of triangular plaquettes and dots then becomes indistinguishable from a perfectly periodic arrangement of the same. In the same spirit, as done in the earlier DD case, we have examined the overlap of the LDOS spectrum of the lattices comprising of $\alpha, \beta \gamma$ and $\beta \delta \gamma$ clusters. The spectrum of each individual periodic system has a common region of overlap again between $E=\epsilon \pm 2 t$ as soon as one sets $\Phi=\Phi_{0} / 4$ and $\lambda=t / \sqrt{2}$. Thus, as before, we
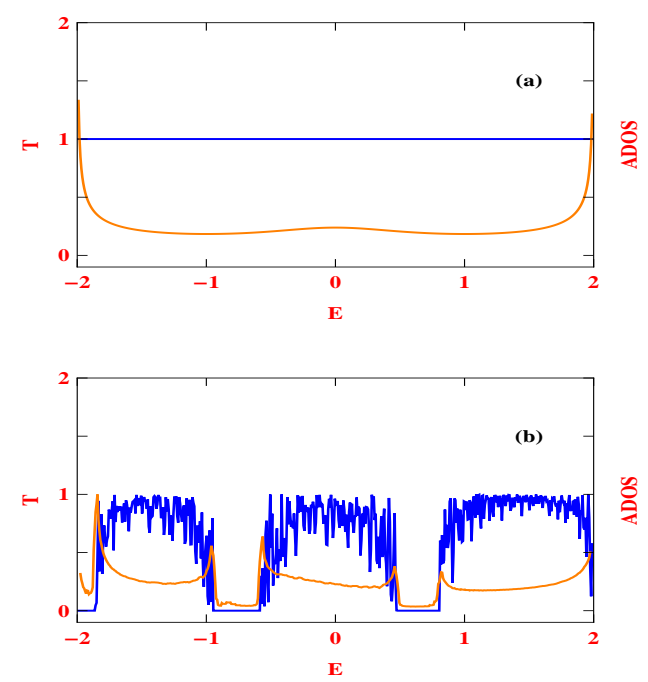

Fig. 6: (Color online). Transmission probability $T$ (blue color) and ADOS (orange color) as a function of energy $E$ for an array of 150 triangles and 150 dots. We have chosen $\epsilon=0, t=1$, and $\lambda=1 / \sqrt{2}$. (a) $\Phi=0.25 \Phi_{0}$, the resonant case, and (b) $\Phi=0.15 \Phi_{0}$, the off-resonance case. The lead parameters are same as in the other figures.

expect a continuum of extended states and perfect transmission irrespective of energy in this region as long as the resonance conditions are satisfied. The important distinction with the DD case is that, now we are able to fine-tune 
the transport in a disordered TD array by dint of an $e x$ ternal magnetic field - a fact that might be useful from the standpoint of designing electronic devices.

The ADOS and the transmission spectrum in the resonant and off-resonant conditions are displayed in fig. 6 for a system of 150 triangles and 150 dots placed randomly along a line. The ballistic character of the transmission coefficient in part (a) of the figure is perfectly in accord with the analysis presented here. In the off-resonance condition we again have sub-bands of high transmittivity. The central band still remains continuous around $E=\epsilon$. The reason is again attributed to the fact that at $\Phi=\Phi_{0} / 4$ the commutivity may also be achieved by setting $E=\epsilon$. This makes the central band survive for a finite sized system even when we deviate from the resonance condition.

$$
* * *
$$

Biplab Pal would like to thank DST, India for providing financial assistance through an INSPIRE Fellowship (IF110078). Santanu K. Maiti is thankful to Abraham Nitzan for stimulating discussions, and gratefully acknowledges the hospitality at the School of Chemistry, Tel Aviv University where part of the computation was done.

\section{REFERENCES}

[1] Anderson P. W., Phys. Rev., 109 (1958) 1492.

[2] Kramer B. and MacKinnon A., Rep. Prog. Phys., 56 (1993) 1469.

[3] Abrahams E., Anderson P. W., Licciardello D. C. and Ramakrishnan T. V., Phys. Rev. Lett., 42 (1979) 673.

[4] Yablonovitch E., Phys. Rev. Lett., 58 (1987) 2059.

[5] John S., Phys. Rev. Lett., 58 (1987) 2486.

[6] Montero De Espinosa F. R., Jiménez E. and Torres M., Phys. Rev. Lett., 80 (1998) 1208.

[7] Vasseur J. O., Deymier P. A., Frantziskonis G., Hong G., Djafari-Rouhani B. and Dobrzynski L., J. Phys.: Condens. Matter, 10 (1998) 6051.

[8] Tao A., Sinsermsuksaul P. and Yang P., Nature Nanotechnology, 2 (2007) 435.

[9] Christ A., Ekinci Y., Solak H. H., Gippius N. A., Tikhodeev S. G. and Martin O. J. F., Phys. Rev. B, 76 (2007) 201405(R).

[10] Barinov I. O., Alodzhants A. P. and Arakelian S. M., Quantum Electronics, 39 (2009) 685.

[11] Grochol M. and Piermarocchi C., Phys. Rev. B, 78 (2008) 035323.

[12] Damski B., Zakrzewski J., Santos L., Zoller P. and Lewenstein M., Phys. Rev. Lett., 91 (2003) 080403.

[13] Billy J., Josse V., Zuo Z., Bernard A., Hambrecht B., Lugan P., Clément D., Sanchez-Palencia L., Bouyer P. and Aspect A., Nature (London), 453 (2008) 891.

[14] Roati G., D'Errico C., Fallani L., Fattori M., Fort C., Zaccanti M., Modugno G., Modugno M. and InGuscio M., Nature (London), 453 (2008) 895.

[15] Römer R. A. and Schulz-BAldes H., Europhys. Lett., 68 (2004) 247.
[16] Eilmes A., Römer R. A. and Schreiber M., Physica $B, 296$ (2001) 46.

[17] Rodríguez A., J. Phys. A: Math. Gen., 39 (2006) 14303.

[18] Rodriguez A., Vasquez L. J. and Römer R. A., Phys. Rev. B, 78 (2008) 195107.

[19] Rodriguez A., Vasquez L. J., Slevin K. and Römer R. A., Phys. Rev. B, 84 (2011) 134209.

[20] Pinski S. D., Schirmacher W. and Römer R. A., Europhys. Lett., 97 (2012) 16007.

[21] Deych L. I., Lisyansky A. A. and Altshuler B. L., Phys. Rev. Lett., 84 (2000) 2678.

[22] Kantelhardt J. W. and Bunde A., Phys. Rev. B, 66 (2002) 035118.

[23] Titov M. and Schomerus H., Phys. Rev. Lett., 95 (2005) 126602.

[24] Mohanty P. and WebB R. A., Phys. Rev. Lett., 88 (2002) 146601.

[25] Dunlap D. H., Wu H-L. and Phillips P. W., Phys. Rev. Lett., 65 (1990) 88.

[26] De Moura F. A. B. F. and Lyra M. L., Phys. Rev. Lett., 81 (1998) 3735.

[27] Sil S., Maiti S. K. and Chakrabarti A., Phys. Rev. $B, 78$ (2008) 113103.

[28] Bellani V., Diez E., Hey R., Toni L., Tarricone L., Parravicini G. B., Domínguez-Adame F. and GómezAlcalá R., Phys. Rev. Lett., 82 (1999) 2159.

[29] Kuhl U., Izrailev F. M., Krokhin A. A. and Stöckmann H.-J., Appl. Phys. Lett., 77 (2000) 633.

[30] Rodriguez A., Chakrabarti A. and Römer R. A., Phys. Rev. B, 86 (2012) 085119.

[31] Rodríguez A. and Cerveró J. M., Phys. Rev. B, 72 (2005) 193312.

[32] Gómez I., Domínguez-Adame F. and Diez E., Physica $B, 324$ (2002) 235

[33] Rodríguez A. and Cerveró J. M., Phys. Rev. B, 74 (2006) 104201.

[34] DatTA S., Electronic transport in mesoscopic systems (Cambridge University Press, Cambridge) 1995 